\title{
Scaling of Shear Rheology of Concentrated Charged Colloidal Suspensions across Glass Transition
}

\author{
Bin Wu, ${ }^{1}$ Takuya Iwashita, ${ }^{2}$ and Wei-Ren $\mathrm{Chen}^{3}$
}

${ }^{1}$ Key Laboratory of Beam Technology of Ministry of Education, College of Nuclear Science and Technology, Beijing Normal University, Beijing 100875, China

${ }^{2}$ Department of Integrated Science and Technology, Oita University, Oita 870-1192, Japan

${ }^{3}$ Neutron Scattering Division, Oak Ridge National Laboratory, Oak Ridge, Tennessee 37831, United States

1. Scaling analyses of rheological data for $\Phi=0.4$ and 0.375
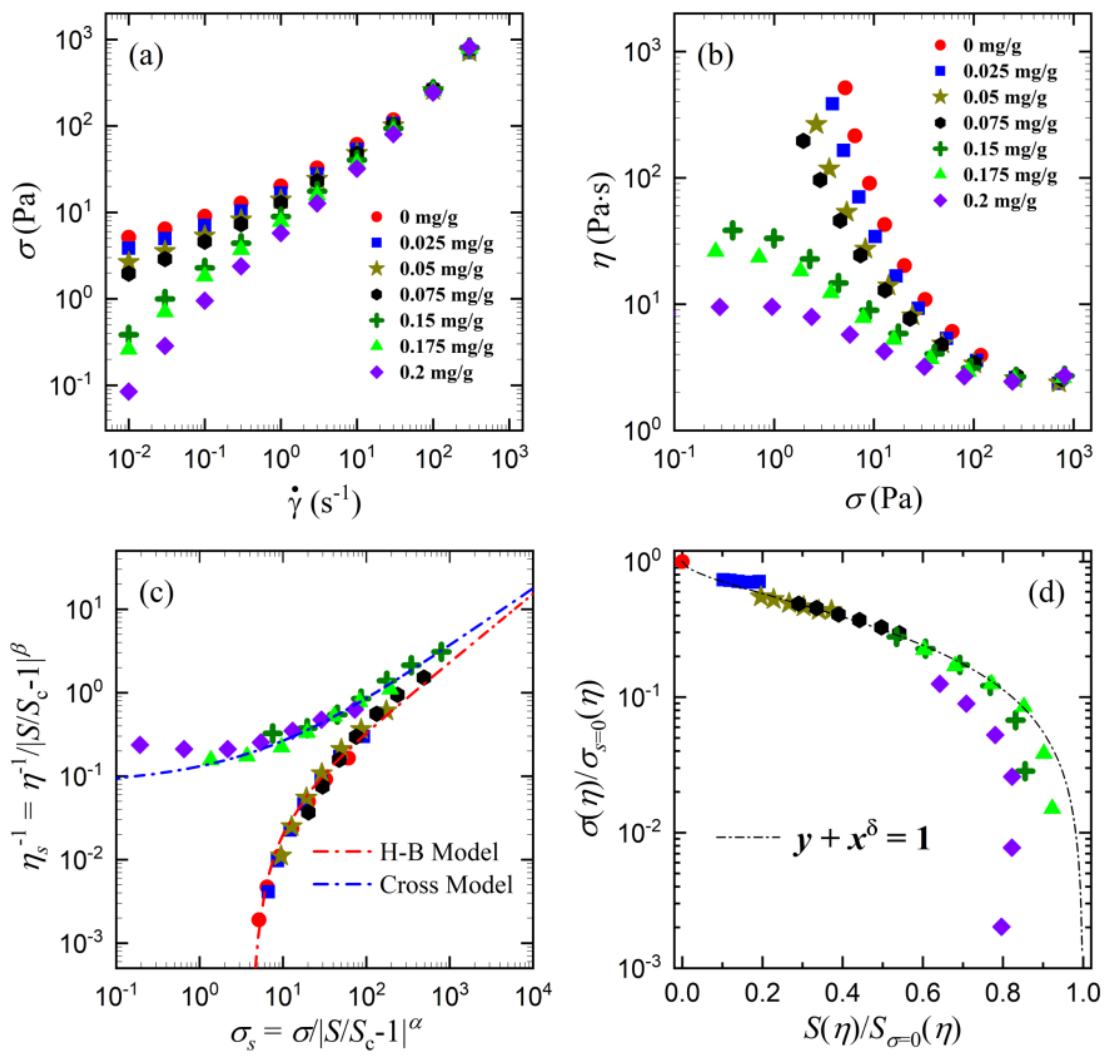

Figure S1 Scaling analyses on $\Phi=0.4$ set of suspensions. Panel (a) and (b) illustrate the measured flow curves, where different symbols represent varying salinity; panel (c) demonstrates the applicability of critical scaling, where the key parameters are $S_{\mathrm{c}}=0.12 \mathrm{mg} / \mathrm{g}, \alpha=2.30$, and $\beta=$ 1.95; panel (d) shows the applicability of isoviscosity scaling, where the exponent $\delta$ in the master curve is 0.54 . 
Table S1 The adjustable parameters of the Herschel-Bulkley and Cross equations determined from modeling the lower and upper branches of critical scaling for $\Phi=0.4$ set of samples, respectively.

\begin{tabular}{|c|c|c|c|}
\hline Herschel-Bulkley & $\sigma_{S Y}(\mathrm{~Pa})$ & $K$ & $v$ \\
\cline { 2 - 4 } & 4.19 & 14.10 & 0.55 \\
\hline \multirow{2}{*}{ Cross } & $\eta_{S N}(\mathrm{~Pa} \cdot \mathrm{s})$ & $C$ & $\kappa$ \\
\cline { 2 - 4 } & 13.22 & 0.13 & 0.41 \\
\hline
\end{tabular}
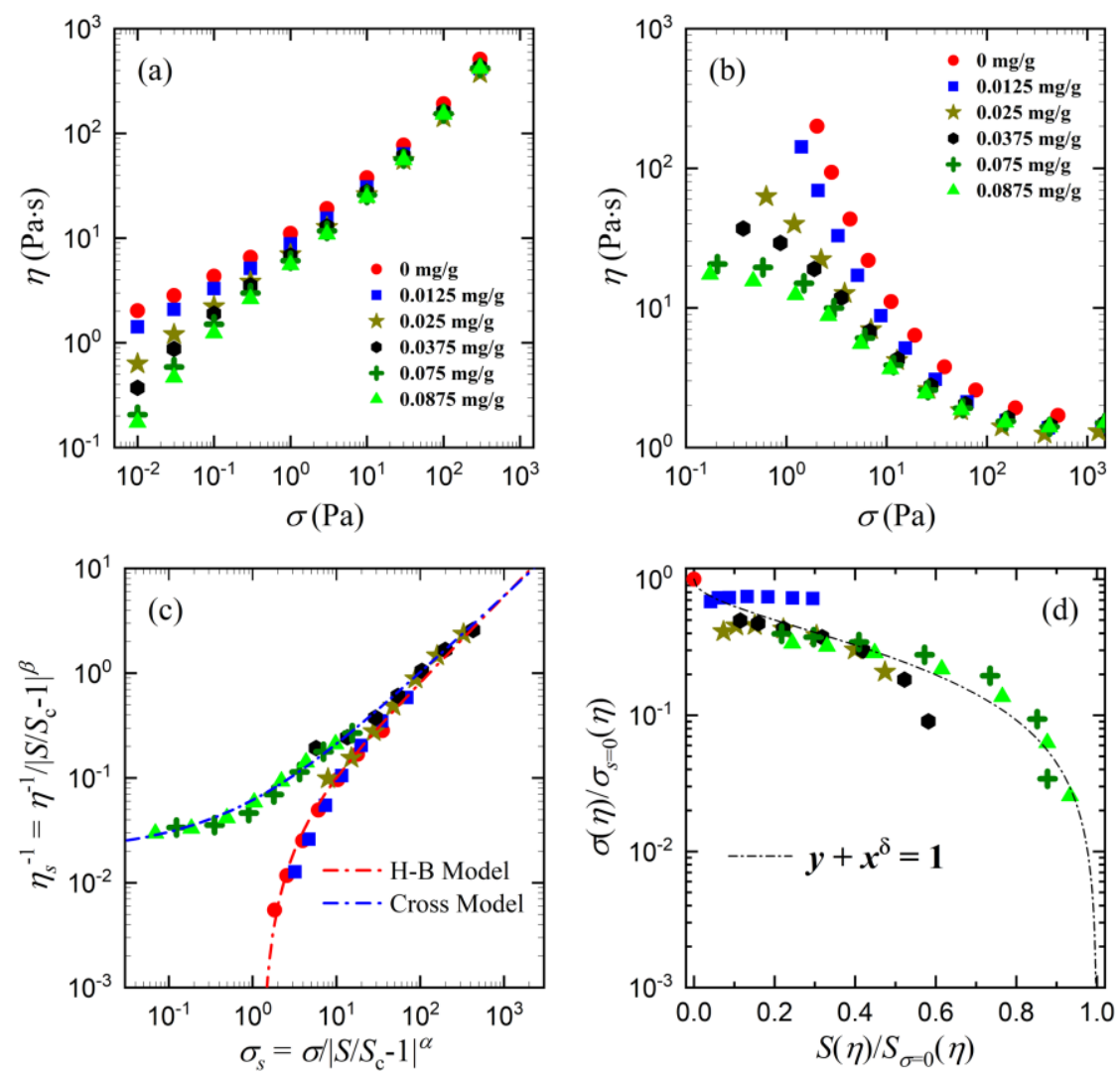

Figure S2 Scaling analyses on $\Phi=0.375$ set of suspensions. Panels (a) and (b) illustrate the measured flow curves, where different symbols represent varying salinity; panel (c) demonstrates the applicability of critical scaling, where the key parameters are $S_{\mathrm{c}}=0.032 \mathrm{mg} / \mathrm{g}, \alpha=1.62$, and $\beta$ 
$=1.17$; panel (d) shows the applicability of isoviscosity scaling, where the exponent $\delta$ in the master curve is 0.43 .

Table S2 The adjustable parameters of the Herschel-Bulkley and Cross equations determined from modeling the lower and upper branches of critical scaling for $\Phi=0.375$ set of samples, respectively.

\begin{tabular}{|c|c|c|c|}
\hline \multirow{2}{*}{ Herschel-Bulkley } & $\sigma_{S Y}(\mathrm{~Pa})$ & $K$ & $v$ \\
\cline { 2 - 4 } & 1.24 & 8.77 & 0.55 \\
\hline \multirow{2}{*}{ Cross } & $\eta_{S N}(\mathrm{~Pa} \cdot \mathrm{s})$ & $C$ & $\kappa$ \\
\cline { 2 - 4 } & 53.78 & 0.14 & 0.43 \\
\hline
\end{tabular}

2. The dependence of shear stress normalized by thermal stress on Péclet number and salt concentration for $\Phi=0.425$

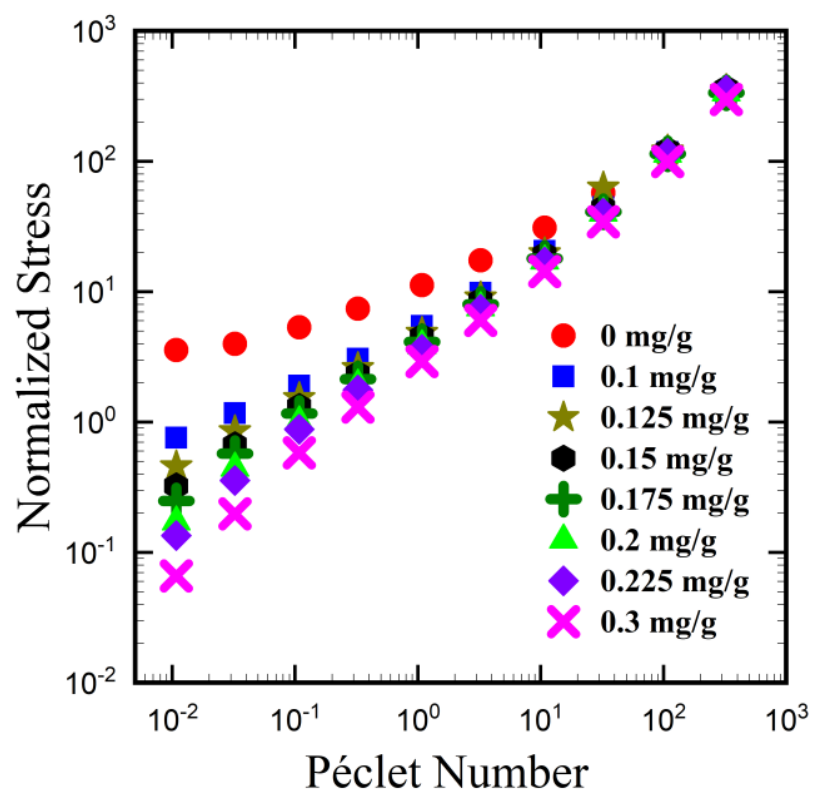

Figure S3 Normalized stress (i.e., shear stress divided by thermal stress) versus Péclet number for $\Phi=0.425$ set of suspensions. 
3. Rescaling of shear stress and shear rate for $\Phi=0.425$

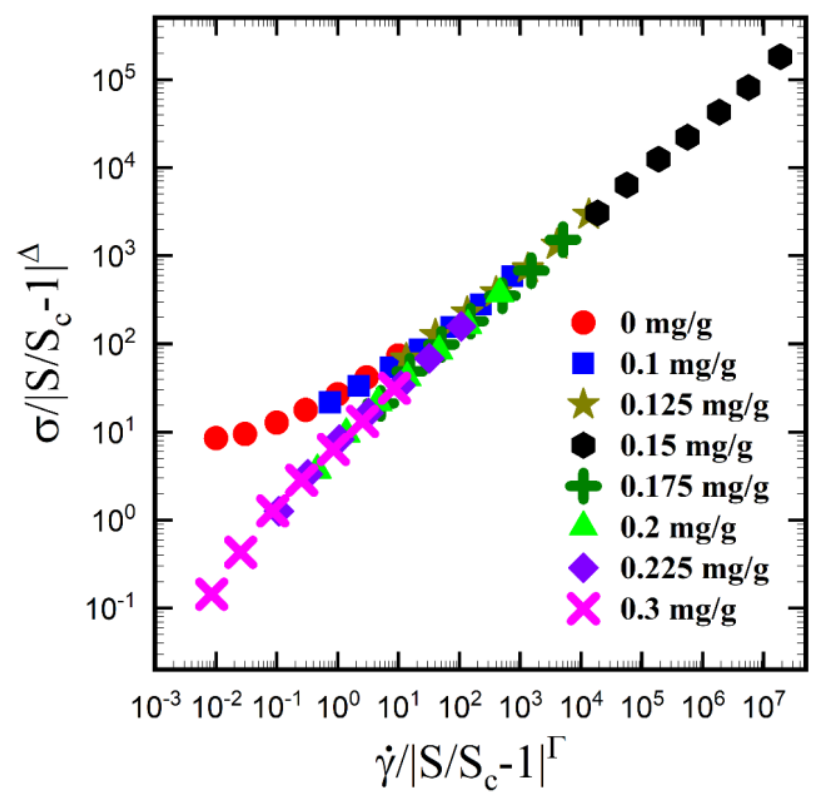

Figure S4 Rescaling the shear stress versus shear rate flow curves for $\Phi=0.425$ set of suspensions, where the key parameters are $S_{\mathrm{c}}=0.1465 \mathrm{mg} / \mathrm{g}, \Gamma=3.78$, and $\Delta=2.17$. Note that the exponents are very close to those determined by Bonn et. al. (Phys. Rev. E. 92, 012305 (2015)), where $\Gamma=$ 3.84 and $\Delta=2.13$ have been reported. 
4. Contour plots of isoviscosity lines for $\Phi=\mathbf{0 . 4 2 5}$

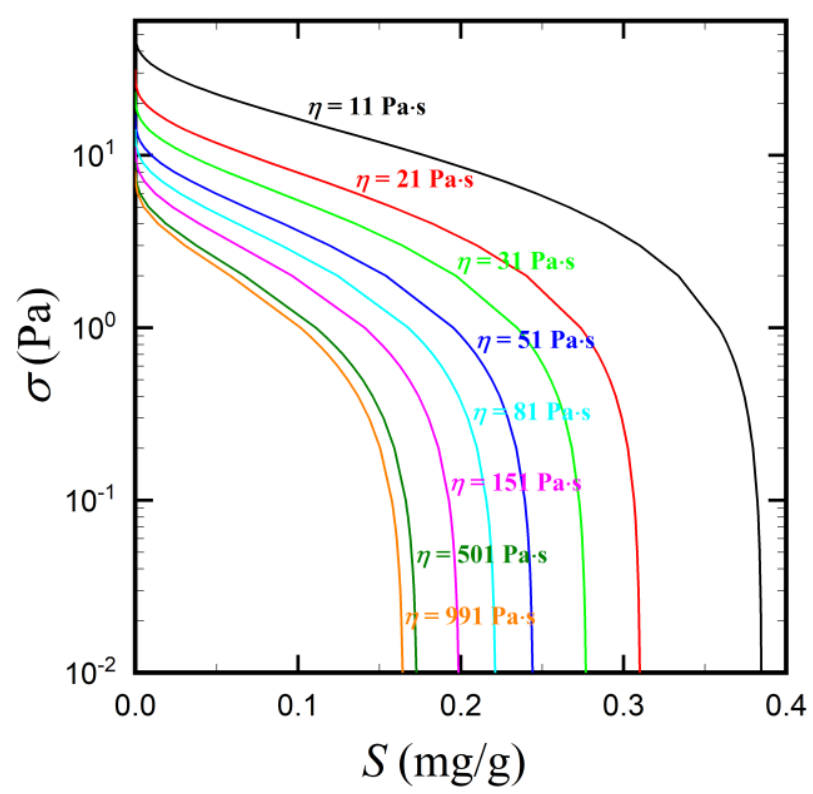

Figure S5 Contour plots for $\Phi=0.425$ set of suspensions based on the isoviscosity scaling relation. 